\title{
Achievements and Open Issues in the Determination of Polarized Parton Distribution Functions
}

\author{
Emanuele R. Nocera \\ Dipartimento di Fisica, Università di Genova, Via Dodecaneso, 33 \\ I-16146 Genova, Italy \\ emanuele.nocera@edu.unige.it
}

Published 29 February 2016

\begin{abstract}
I review the current status of the determination of helicity-dependent, or polarized, parton distribution functions from a comprehensive analysis of experimental data in perturbative quantum chromodynamics. I illustrate the latest achievements driven by new measurements in polarized proton-proton collisions at the Relativistic Heavy Ion Collider, namely the first evidence of a sizable polarized light sea quark asymmetry and of a positive polarized gluon distribution in the proton. I discuss which are the open issues in the determination of polarized distributions, and how these may be addressed in the future by ongoing, planned and proposed experimental programs.
\end{abstract}

Keywords: Polarized parton distribution functions; nucleon spin structure.

PACS numbers: $13.88 .+\mathrm{e}, 13.85 . \mathrm{Ni}$

Understanding how the nucleon spin is built up from the spin of quarks and gluons and their orbital angular momentum - is one of the most challenging goals in hadron physics. ${ }^{1}$ The way to decompose the one-half nucleon spin into gauge-invariant, physically meaningful and measurable quantities has raised a major controversy in the last years, partly clarified only very recently. ${ }^{2}$ For instance, according to the Jaffe and Manohar helicity sum rule ${ }^{3}$

$$
\frac{1}{2}=\frac{1}{2} \Delta \Sigma\left(\mu^{2}\right)+\Delta G\left(\mu^{2}\right)+\mathcal{L}_{q}\left(\mu^{2}\right)+\mathcal{L}_{g}\left(\mu^{2}\right),
$$

contributions to the proton spin are explicit split into quark and gluon spin, $\Delta \Sigma$ and $\Delta G$, and quark and gluon orbital angular momentum, $\mathcal{L}_{q}$ and $\mathcal{L}_{g}$. Each term in the r.h.s. of Eq. (1) allows for a proper field-theoretic definition in Quantum Chromodynamics (QCD), ${ }^{4}$ and is usually probed by measuring spin asymmetries, i.e. differences of cross sections with opposite polarizations of initial-state particles, that arise

This is an Open Access article published by World Scientific Publishing Company. It is distributed under the terms of the Creative Commons Attribution 3.0 (CC-BY) License. Further distribution of this work is permitted, provided the original work is properly cited. 
in a large variety of high-energy, large-momentum-transfer processes. ${ }^{5}$ Following factorization, ${ }^{6}$ these are described as a convolution between a short-distance part (that contains information on the hard interactions of partons in the form of processdependent kernels) and a long-distance part (that contains information on the spin structure of the nucleon in the form of universal parton distributions). The former can be computed in perturbative QCD; the latter should be determined from experimental data; both depend on the factorization scheme and scale $\mu$.

In this write-up, I review recent progress in the determination of helicitydependent, or longitudinally polarized, Parton Distributions Functions (PDFs)

$$
\Delta f\left(x, \mu^{2}\right) \equiv f^{\uparrow}\left(x, \mu^{2}\right)-f^{\downarrow}\left(x, \mu^{2}\right), \quad f=u, \bar{u}, d, \bar{d}, s, \bar{s}, g
$$

defined as the momentum densities of partons with spin aligned along $\left({ }^{\uparrow}\right)$ or opposite $\left({ }^{\downarrow}\right)$ the polarization direction of the parent nucleon. Polarized PDFs encode the spin structure of the nucleon, since these are related to the first two terms in Eq. (1):

$$
\Delta \Sigma\left(\mu^{2}\right)=\sum_{q=u, d, s} \int_{0}^{1} d x\left[\Delta q\left(x, \mu^{2}\right)+\Delta \bar{q}\left(x, \mu^{2}\right)\right] \quad \Delta G\left(\mu^{2}\right)=\int_{0}^{1} d x \Delta g\left(x, \mu^{2}\right) .
$$

The dependence of the PDFs on $x$, the momentum fraction of the proton carried by the parton, is genuinely nonperturbative, and, as such, must be inferred from data. However, because of their modest quantity and quality, these are usually supplemented with some theoretical constraints in order to achieve a meaningful PDF determination. First, PDFs must lead to positive cross sections: at leading order (LO), this implies that polarized PDFs are bounded by their unpolarized counterparts $^{\mathrm{a}},\left|\Delta f\left(x, \mu^{2}\right)\right| \leq f\left(x, \mu^{2}\right)$. Second, polarized PDFs must be integrable: this corresponds to the assumption that the nucleon matrix element of the axial current for each flavor is finite. Third, it follows from SU(2) and $\mathrm{SU}(3)$ flavor symmetry that the first moments of the nonsinglet $\mathcal{C}$-even PDF combinations, $\Delta T_{3}=\Delta u^{+}-\Delta d^{+}$ and $\Delta T_{8}=\Delta u^{+}+\Delta d^{+}-2 \Delta s^{+}$(where $\Delta q^{+}=\Delta q+\Delta \bar{q}, q=u, d, s$ ), are related to the baryon octet $\beta$-decay constants, whose values are well measured: ${ }^{8}$

$$
a_{3}=\int_{0}^{1} d x \Delta T_{3}=1.2701 \pm 0.0025 \quad a_{8}=\int_{0}^{1} d x \Delta T_{8}=0.585 \pm 0.025 .
$$

The dependence of the PDFs on $\mu$ is perturbative. This obeys DokshitzerGribov-Lipatov-Altarelli-Parisi (DGLAP) evolution equations, ${ }^{9}$ and has been computed up to next-to-next-to-leading order (NNLO) recently. ${ }^{10}$ In principle, a determination of polarized PDFs at NNLO is then possible, even though based on inclusive DIS data alone (coefficient functions are known at NNLO only for the polarized DIS structure function $g_{1}{ }^{11}$ ) and in a fixed-flavor-number scheme (nontrivial matching coefficients for the strong coupling $\alpha_{s}$ and PDFs are not known at

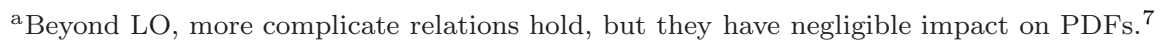


NNLO). In practice, the impact of NNLO corrections is smaller than the current experimental uncertainties on data, hence moving from next-to-leading order (NLO) to NNLO determinations of polarized PDFs is neither convenient nor needed so far.

Several determinations of polarized PDFs of the proton (up to NLO and mostly in the $\overline{\mathrm{MS}}$ factorization scheme) are presently available, aiming at unveiling how much large and uncertain are $\Delta \Sigma$ and $\Delta G$, Eq. (3). Above all, they differ among each others for the procedure used to determine PDFs from data and for the included data sets (for details, see e.g. Chap. 3 in Ref. 12). Both are a source of uncertainty, but the former may be elusive: in this respect, the NNPDF collaboration has developed a methodology to reduce and keep it under control as much as possible. This is based on cutting-edge statistical tools, including Monte Carlo sampling for error propagation, neural networks for PDF parametrization, and closure tests for explicit characterization of procedural uncertainties. ${ }^{13}$

The bulk of the experimental information on polarized PDFs comes from neutralcurrent inclusive and semi-inclusive Deep-Inelastic Scattering (DIS and SIDIS) with charged lepton beams and nuclear targets. Because of the way the corresponding observables factorize, inclusive DIS data constrain the total quark combinations $\Delta q^{+}$, while SIDIS data, with identified pions or kaons in the final state, constrain individual quark and antiquark flavors. In principle, both DIS and SIDIS data would constrain the gluon distribution $\Delta g$ via scaling violations, but in practice their effect is rather weak because of the small $Q^{2}$ range covered. One- or two-hadron and open-charm production may provide a direct handle on $\Delta g$, but the available data have almost no effect on it because of their limited statistics. ${ }^{14}$

Beside DIS and SIDIS fixed-target data, a significant amount of data from longitudinally polarized proton-proton $(p p)$ collisions at the Relativistic Heavy Ion Collider (RHIC) have become available recently. ${ }^{15}$ Motivated by the interest in studying the effects of this piece of experimental information, two new global analyses of polarized PDFs have been carried out in 2014, DSSV14 ${ }^{16}$ and NNPDFpol1.1. ${ }^{17}$ These upgrade the corresponding previous analyses, DSSV08 ${ }^{18}$ and NNPDFpol1.0, ${ }^{19}$ with data respectively on double-spin asymmetries for inclusive jet production ${ }^{20}$ and $\pi^{0}$ production $^{21 \mathrm{~b}}$, and on double-spin asymmetries for high- $p_{T}$ inclusive jet production $^{20,22,23}$ and single-spin asymmetries for $W^{ \pm}$production. $^{24}$

The effect of these data on the polarized PDFs of the proton is twofold.

- Because of the chiral nature of the weak interactions, both (parity-violating) single-spin and (parity-conserving) double-spin asymmetries for $W^{ \pm}$boson production are sensitive to the flavor decomposition of polarized quark and antiquark distributions. $^{25}$ The 2012 STAR data sets ${ }^{24}$ included in NNPDFpol1. 1 are the first of this kind to provide a substantial impact on light sea antiquark polarizations. Specifically, it was found ${ }^{17}$ that they favor a positive $\Delta \bar{u}$ distribution and a negative $\Delta \bar{d}$ distribution, with $|\Delta \bar{d}|>|\Delta \bar{u}|$. First evidence of broken flavor symmetry

${ }^{\mathrm{b}}$ Preliminary RHIC results included in Ref. 18 have been replaced in Ref. 16 with final results. 
for polarized light sea quarks is then emerging; the size of such a breaking is quantified by the asymmetry $\Delta \bar{u}-\Delta \bar{d}$, which turned out to be roughly as large as its unpolarized counterpart (in absolute value), though much more uncertain. ${ }^{26}$ Even within this uncertainty, polarized and unpolarized light sea quark asymmetries show opposite sings, with the polarized being definitely positive. This is a peculiar feature driven by RHIC data, which extend the information provided by SIDIS data. This result starts to discriminate between different models of nucleon structure, see left panel of Fig. 1: specifically, some meson-cloud (MC) models are disfavored, while a more accurate experimental information is needed to establish whether chiral quark-soliton (CQS), Pauli-blocking (PB) or statistical (ST) models are preferred (a description of these models can be found in Ref. 27).

- Because of the dominance of gluon-gluon and quark-gluon initiated subprocesses in the kinematic range accessed by RHIC, $0.05 \lesssim x \lesssim 0.2$, double-spin asymmetries for jet and $\pi^{0}$ production are directly sensitive to the gluon polarization in the proton. ${ }^{28}$ The 2009 STAR and PHENIX data sets, ${ }^{20,21}$ included in DSSV14 and NNPDFpol1.1, are the first of this kind to provide first evidence of a sizable, positive gluon polarization in the proton. A comparison of the gluon PDF in the two PDF sets is displayed in Fig. 1 (right panel). Comparable results, both central values and uncertainties, are found in the $x$ region covered by RHIC data. The agreement between the two analyses is optimal in the range $0.08 \leq x \leq 0.2$, where the dominant experimental information comes from jet data; a slightly smaller central value is found in the DSSV14 analysis, in comparison to the NNPDFpol1.1,
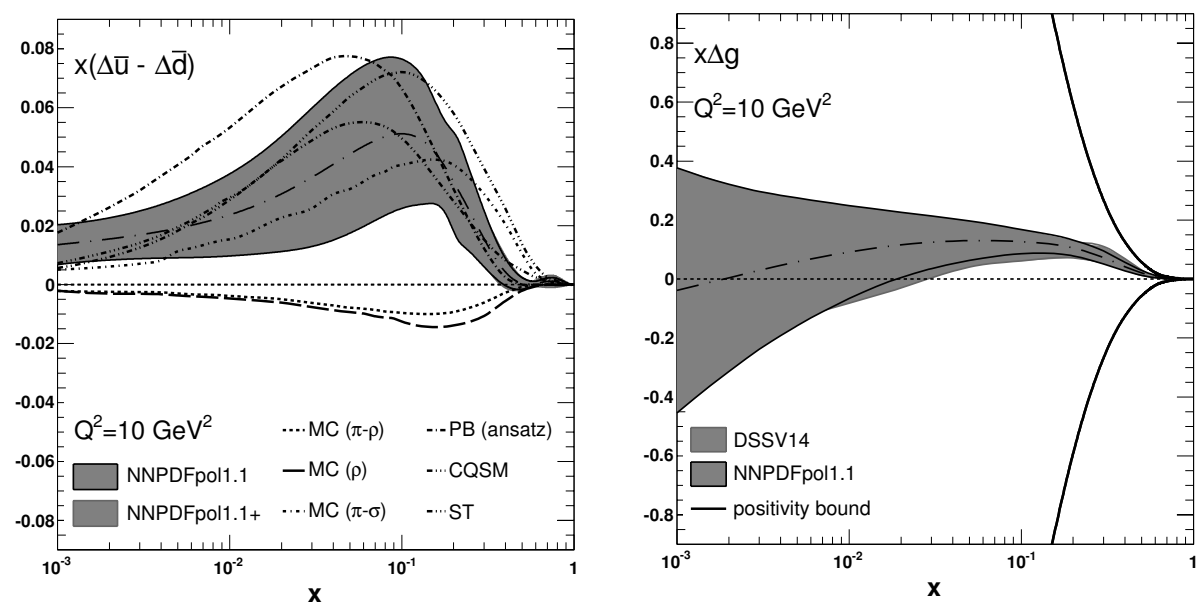

Figure 1. (Left panel) The polarized light sea quark asymmetry $x(\Delta \bar{u}-\Delta \bar{d})$ computed with NNPDFpol1.1 and NNPDFpol1.1+ (supplemented with pseudodata for $W^{ \pm}$production at RHIC) PDF sets at $Q^{2}=10 \mathrm{GeV}^{2}$, compared to expectations from various models of nucleon structure. ${ }^{27}$ (Right panel) The polarized gluon distribution $\Delta g$ from the DSSV14 ${ }^{16}$ and NNPDFpol1.1 $1^{26}$ PDF sets at $Q^{2}=10 \mathrm{GeV}^{2}$. 
in the range $0.05 \leq x \leq 0.08$, where the dominant experimental information comes from $\pi^{0}$ production data. Indeed, these are included in DSSV14 but are not in NNPDFpol1.1. Nevertheless, best fits lie well within each other error bands, though NNPDF uncertainties tend to be larger than DSSV14 uncertainties outside the region covered by RHIC data. Very well compatible values of the integral of $\Delta g$, Eq. (3), truncated over the interval $0.05 \leq x \leq 1$, are found: at $Q^{2}=10$ $\mathrm{GeV}^{2}$, this is $0.20_{-0.07}^{+0.06}$ for DSSV14, ${ }^{16}$ and $0.23 \pm 0.06$ for NNPDFpol1.1. ${ }^{17}$

Beside RHIC data, some new data by the COMPASS experiment have been included in DSSV14 and NNPDFpol1.1, respectively new DIS and SIDIS data ${ }^{29,30}$ and open-charm leptoproduction data. ${ }^{31}$ However, they turned out to have a limited impact on PDFs. ${ }^{16,17}$ Overall, both DSSV14 and NNPDFpol1.1 PDF determinations are state-of-the-art in the inclusion of the available experimental information. The data sets in the two analyses differ between each other only for fixed-target SIDIS and RHIC $\pi^{0}$ production measurements, included in DSSV14, but not in NNPDFpol1.1. The information brought in by these data is complementary to that provided by RHIC $W^{ \pm}$production and inclusive jet production data respectively, but this is less constraining. ${ }^{17}$ These data sets were not included in the NNPDFpol1.1 analysis because fragmentation functions (FFs) enter the factorized expression of the corresponding observables: since FFs are nonperturbative objects on the same footing as PDFs, they are likely to introduce an additional source of bias in the PDF determination. The NNPDF methodology aims at reducing this bias as much as possible, hence the inclusion of these data would require the consistent determination of FFs within the NNPDF methodology, which is not yet available.

Despite the achievements described above, the lack of experimental data over a wide range of $x$ and $Q^{2}$ values seriously limits the accuracy with which polarized PDFs can be determined. Several issues in our knowledge of the nucleon longitudinal spin structure are hence left completely open, as summarized below.

- The contribution of quarks, antiquarks and gluons to the nucleon spin, as quantified by their first moments, Eq. (3), is shadowed by large uncertainties coming from extrapolation into the small- $x$ region $\left(x \lesssim 10^{-3}\right)$, not covered by experimental data. In order to illustrate the situation, the running integrals of singlet and gluon distributions (i.e. the quantities in Eq. (3) evaluated as a function of the lower limit of integration $x_{\text {min }}$ ) are displayed in Fig. 2 at $Q^{2}=10 \mathrm{GeV}^{2}$. It is apparent that, as $x_{\text {min }}$ decreases, the uncertainty on the integrals increases up to a size that prevents from any firm conclusion on their contribution to Eq. (1).

- A precision test of the Bjorken sum rule ${ }^{32}$ is presently not achievable within the accuracy of available data: indeed, a largely uncertain, and potentially substantial, contribution to it may arise in the small- $x$ region, $x \lesssim 10^{-3} \cdot{ }^{19}$ As a consequence, a determination of $\alpha_{s}$ from the Bjorken sum rule is not competitive.

- Fairly significant violations of $\mathrm{SU}(3)$ symmetry are advocated in the literature (see e.g. Ref. 33 for a review). In this case, an uncertainty on the octet axial charge, larger up to $30 \%$ than its experimental value, Eq. (4), is found. ${ }^{34}$ It was 

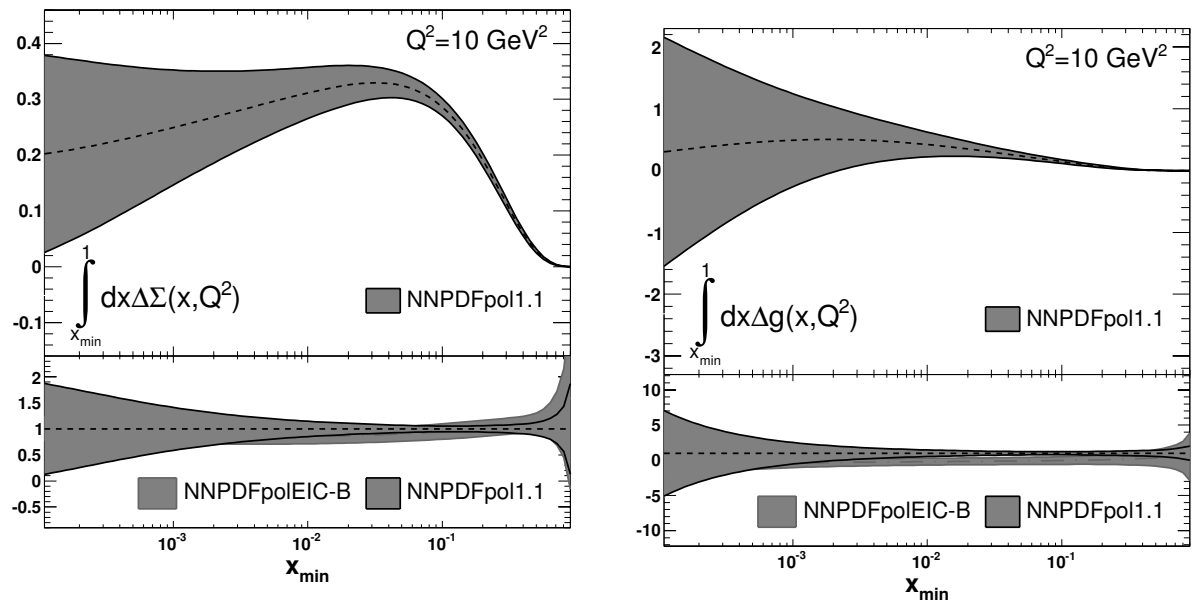

Figure 2. The running integral $\int_{x_{\min }}^{1} d x \Delta f\left(x, Q^{2}\right), f=\Sigma, g$, from NNPDFpol1. $1^{17}$ at $Q^{2}=10$ $\mathrm{GeV}^{2}$ (upper panels), and their ratio to NNPDFpolEIC-B ${ }^{37}$ based on EIC pseudodata (lower panels).

shown ${ }^{19}$ that a more conservative estimate of the uncertainty on $a_{8}$ has a limited impact on the behavior of polarized PDFs in a global analysis. Nevertheless, if the octet sum rule in Eq. (4) were not imposed at all, data alone would not be sufficiently accurate either to constrain $\Delta T_{8}$ or to test potential $\mathrm{SU}(3)$ violations.

- Inclusive DIS data, together with nonsinglet axial couplings, Eq. (4), and kaontagged SIDIS data provide the sole constraint on the total strange distribution $\Delta s^{+}$. No experimental information is available on individual $\Delta s$ and $\Delta \bar{s}$ PDFs, and $\Delta s=\Delta \bar{s}$ is often assumed for phenomenological purposes. Mutually consistent, sizable, negative values of the first moment of $\Delta s^{+}$are found in both DSSV $^{16,18}$ and NNPDF ${ }^{17,19}$ analyses, though they arise from rather different shapes of $\Delta s^{+}$. This discrepancy may considerably depend on the kaon FF used to analyze SIDIS data, ${ }^{35}$ which are included in the DSSV determinations and are not in the NNPDF determinations. Further measurements of spin-dependent kaon production cross section in SIDIS and an improved determination of the kaon FF are needed to make any definitive conclusion.

- Several models of nucleon structure have been developed, aiming at predicting the polarized PDF behavior at small and large $x$. In order to test whether data favor or unfavor them, computations of spin-dependent observables based on these models should be compared to the corresponding expectations based on a global analysis of polarized PDFs. Unfortunately, only a limited number of models are clearly incompatible with the predictions based on DSSV14 and NNPDFpol1.1. ${ }^{36}$ More abundant and more accurate data at small and large $x$ would be needed to discriminate among the other models. 
A campaign of ongoing, planned and proposed experiments would address these issues in the future: some short- and long-term prospects are discusssed in the sequel.

- The COMPASS experiment at CERN is about completing its experimental program dedicated to the longitudinal spin structure of the nucleon. It will provide additional high-precision results for the inclusive DIS structure function of the proton $g_{1}^{p}{ }^{38}$ However, these are expected to be of limited impact in narrowing the small- $x$ extrapolation uncertainty on the full first moments, Eq. (3), since they will extend only down to $x \sim 0.004$.

- The CLAS and Hall-A experiments at JLAB have recently presented a large amount of high-precision data respectively on the ratio of polarized to unpolarized proton and deuteron structure functions, $g_{1}^{p, d} / F_{1}^{p, d}, 39$ and on the proton and neutron spin photoabsorption asymmetry, $A_{1}^{p, n}$. ${ }^{40}$ Since these data are taken in a kinematic region (large $x$, small $Q^{2}$ ) where dynamic higher-twist contributions to the Wilson expansion of $g_{1}$ and resummation effects may be relevant, ${ }^{41,42}$ their inclusion in a global analysis of polarized PDFs is highly nontrivial, and not yet performed. The kinematic reach of the existing JLAB data is expected to extend to twice smaller $x$ as well as to larger $x$ values after the $12 \mathrm{GeV}$ electron beam energy upgrade: ${ }^{43}$ large luminosity and high resolution available will allow for a substantial reduction of PDF uncertainties in the medium-to-large $x$ region.

- The STAR and PHENIX experiments at RHIC are expected to provide highimpact results in the near term. ${ }^{15}$ As for $W^{ \pm}$production, much smaller uncertainties on the single-spin asymmetry will be reached thanks to the substantially increased statistics from run-2013. Supplementing NNPDFpol1.1 with $W^{ \pm}$production pseudodata based on projected uncertainties will reduce the uncertainty on the polarized light sea quark asymmetry by a factor two, see Fig. 1 (the PDF determination including pseudodata is labeled NNPDFpol1.1+). As for $\pi^{0}$ and jet production, future RHIC measurements up to run-2015 at center-of-mass energy $\sqrt{s}=510 \mathrm{GeV}$ will be sensitive to the polarized gluon PDF down to $x \sim 10^{-3}$. Inclusion of $\pi^{0}$ and jet production pseudodata based on projected uncertainties in the DSSV14 analysis will reduce the uncertainty on $\Delta g$ by a factor two. ${ }^{15}$ Complementary information on $\Delta g$ will be provided by correlation measurements, e.g. in di-jet and di-hadron production processes.

- A future high-energy, polarized Electron-Ion Collider (EIC) ${ }^{44}$ will likely be the only facility to address all the above questions with the highest precision. The extension of the kinematic reach down to $x \sim 10^{-4}$ and $Q^{2}=10^{4} \mathrm{GeV}^{2}$ will allow for an accurate determination of $\Delta g$ via scaling violations in inclusive DIS, of $\Delta \bar{u}$ and $\Delta \bar{d}$ via inclusive DIS at high $Q^{2}$ mediated by electroweak bosons, and of $\Delta s$ via kaon-tagged SIDIS. Using simulated pseudodata at the eRHIC realization of an EIC, ${ }^{45}$ the impact of some of these measurements has been recently studied. ${ }^{37,46}$ It was found that the running integrals $\Delta \Sigma$ and $\Delta G$ will be 
determined with an accuracy of about respectively $\pm 20 \%$ and $\pm 10 \%$, see Fig. 2 . Would the EIC data confirm the DSSV14 or NNPDFpol1.1 best fit behaviors of $\Delta \Sigma$ and $\Delta G$, see Fig. 2, only a small fraction of the proton spin is expected to come from orbital angular momentum, $\mathcal{L}_{q}+\mathcal{L}_{g}$ in Eq. (1), at $Q^{2}=10 \mathrm{GeV}^{2}$.

\section{Bibliography}

1. S. D. Bass, The Spin structure of the proton (World Scientific, 2007).

2. E. Leader and C. Lorce, Phys.Rept. 541, 163 (2013).

3. R. Jaffe and A. Manohar, Nucl.Phys. B337, 509 (1990).

4. J. Collins, Foundations of perturbative QCD (Cambridge monographs on particle physics, nuclear physics and cosmology, 2011).

5. C. A. Aidala, S. D. Bass, D. Hasch and G. K. Mallot, Rev.Mod.Phys. 85, 655 (2013).

6. J. C. Collins, D. E. Soper and G. F. Sterman, Adv.Ser.Direct.High Energy Phys. 5, 1 (1988), J. C. Collins, Nucl.Phys. B394, 169 (1993).

7. G. Altarelli, S. Forte and G. Ridolfi, Nucl.Phys. B534, 277 (1998).

8. K. Olive et al., Chin.Phys. C38, 090001 (2014).

9. G. Altarelli and G. Parisi, Nucl.Phys. B126, 298 (1977).

10. S. Moch, J. Vermaseren and A. Vogt, Nucl.Phys. B889, 351 (2014).

11. E. Zijlstra and W. van Neerven, Nucl.Phys. B417, 61 (1994).

12. E. R. Nocera, Unbiased spin-dependent parton distribution functions, $\mathrm{PhD}$ thesis, Physics, Astrophysics and Applied Physics PhD School, University of Milan, 2014.

13. R. D. Ball et al. (2014).

14. E. R. Nocera, PoS DIS2013, p. 211 (2013).

15. E.-C. Aschenauer, A. Bazilevsky, M. Diehl, J. Drachenberg, K. O. Eyser et al. (2015).

16. D. de Florian et al., Phys.Rev.Lett. 113, 012001 (2014).

17. E. R. Nocera et al., Nucl.Phys. B887, 276 (2014).

18. D. de Florian et al., Phys.Rev.Lett. 101, 072001 (2008), Phys.Rev. D80, 034030 (2009).

19. R. D. Ball et al., Nucl.Phys. B874, 36 (2013).

20. L. Adamczyk et al. (2014).

21. A. Adare et al., Phys.Rev. D90, 012007 (2014).

22. L. Adamczyk et al., Phys.Rev. D86, 032006 (2012).

23. A. Adare et al., Phys.Rev. D84, 012006 (2011).

24. L. Adamczyk et al., Phys.Rev.Lett. 113, 072301 (2014).

25. C. Bourrely and J. Soffer, Phys.Lett. B314, 132 (1993).

26. E. R. Nocera, PoS DIS2014, 204 (2014).

27. W.-C. Chang and J.-C. Peng, Prog.Part.Nucl.Phys. 79, 95 (2014).

28. C. Bourrely, J. Guillet and J. Soffer, Nucl.Phys. B361, 72 (1991).

29. M. Alekseev et al., Phys.Lett. B690, 466 (2010).

30. M. Alekseev et al., Phys.Lett. B693, 227 (2010).

31. C. Adolph et al., Phys.Rev. D87, 052018 (2013).

32. J. Bjorken, Phys.Rev. 148, 1467 (1966), Phys.Rev. D1, 1376 (1970).

33. N. Cabibbo, E. C. Swallow and R. Winston, Ann.Rev.Nucl.Part.Sci. 53, 39 (2003).

34. R. Flores-Mendieta, E. E. Jenkins and A. V. Manohar, Phys.Rev. D58, 094028 (1998).

35. E. Leader, A. V. Sidorov and D. B. Stamenov, Phys.Rev. D84, 014002 (2011).

36. E. R. Nocera, Phys.Lett. B742, 117 (2015).

37. R. D. Ball et al., Phys.Lett. B728, 524 (2014).

38. M. Wilfert, PoS DIS2014, 206 (2014).

39. Y. Prok et al., Phys.Rev. C90, 025212 (2014).

40. D. Parno et al. (2014). 
41. P. Jimenez-Delgado, A. Accardi and W. Melnitchouk, Phys.Rev. D89, 034025 (2014). 42. D. P. Anderle, F. Ringer and W. Vogelsang, Phys.Rev. D87, 094021 (2013).

43. J. Dudek, R. Ent, R. Essig, K. Kumar, C. Meyer et al., Eur.Phys.J. A48, 187 (2012).

44. A. Accardi, J. Albacete, M. Anselmino, N. Armesto, E. Aschenauer et al. (2012).

45. E. Aschenauer, M. Baker, A. Bazilevsky, K. Boyle, S. Belomestnykh et al. (2014).

46. E. C. Aschenauer et al., Phys.Rev. D86, 054020 (2012), Phys.Rev. D88, 114025 (2013). 